\title{
D meson semileptonic form factors with HISQ valence and sea quarks
}

T. $\operatorname{Primer}^{a}$, A. Bazavov ${ }^{b *}$, C. Bernard ${ }^{c}$, C. DeTar ${ }^{d}$, D. Du ${ }^{e}$, A.X. El-Khadra ${ }^{f}$, E.D. Freeland ${ }^{g}$, E. Gámiz ${ }^{h}$, Steven Gottlieb ${ }^{t}{ }^{b}$, U.M. Heller ${ }^{i}$, J. Komijani ${ }^{j}$, A.S. Kronfeld ${ }^{j, k}$, J. Laiho ${ }^{e}$, P.B. Mackenzie ${ }^{k}$, E.T. Neil ${ }^{l, m}$, J.N. Simone ${ }^{k}$, R.L. Sugar ${ }^{n}$, D. Toussaint ${ }^{a}$, R.S. Van de Water ${ }^{k}$, and R. Zhou ${ }^{k}$

${ }^{a}$ Physics Department, University of Arizona, Tucson, AZ 85721, USA

${ }^{b}$ Department of Physics, Indiana University, Bloomington, IN 47405, USA

${ }^{c}$ Department of Physics, Washington University, St. Louis, MO 63130, USA

${ }^{d}$ Department of Physics and Astronomy, University of Utah, Salt Lake City, UT 84112, USA

e Department of Physics, Syracuse University, Syracuse, NY 13244, USA

${ }^{f}$ Department of Physics, University of Illinois, Urbana, IL 61801, USA

${ }^{g}$ Liberal Arts Department, School of the Art Institute of Chicago, Chicago, IL 60603, USA

${ }^{h}$ CAFPE and Departamento de Física Teórica y del Cosmos, Universidad de Granada, E-18071 Granada, Spain

${ }^{i}$ American Physical Society, One Research Road, Ridge, NY 11961, USA

${ }^{j}$ Institute for Advanced Study, Technische Universität München, 85748 Garching, Germany

${ }^{k}$ Fermi National Accelerator Laboratory, Batavia, IL 60510 USA

${ }^{l}$ Department of Physics, University of Colorado, Boulder, CO 80309, USA

${ }^{m}$ RIKEN-BNL Research Center, Brookhaven National Laboratory, Upton, NY 11973, USA

${ }^{n}$ Department of Physics, University of California, Santa Barbara, CA 93106, USA

Fermilab Lattice and MILC Collaborations

E-mail: sgeindiana.edu, thomas.primer@gmail.com

We present a calculation of the form factors of the $D \rightarrow K l v$ and $D \rightarrow \pi l v$ semileptonic decays at zero momentum transfer, ultimately for the purpose of determining the Cabibbo-KobayashiMaskawa (CKM) matrix elements $\left|V_{c s}\right|$ and $\left|V_{c d}\right|$. This work uses MILC $N_{f}=2+1+1$ configurations with the HISQ action for both sea quarks and valence quarks, including several physical mass ensembles and lattice spacings down to $0.042 \mathrm{fm}$. The calculation is done directly at $q^{2}=0$ by employing twisted boundary conditions to tune the child particle momenta. Results at the physical point and in the continuum limit will be achieved in the final analysis through the use of Heavy-Meson Staggered $\chi$ PT. Here we present our expected error budget and compare with the error of prior calculations.

34th annual International Symposium on Lattice Field Theory

24-30 July 2016

University of Southampton, UK

${ }^{*}$ Current address: Department of Computational Mathematics, Science and Engineering and Department of Physics and Astronomy, Michigan State University, East Lansing, MI 48824, USA

${ }^{\dagger}$ Speaker. 


\section{Introduction}

The CKM matrix describes quark mixing in the Standard Model. The parameters that characterize the matrix must be deduced from a combination of theory and experiment. At the Lagrangian level, these parameters come from Yukawa couplings, and presumably require a more fundamental theory to explain their values. The CKM matrix may also hold the key to finding evidence of physics beyond the Standard Model. A violation of unitarity of the CKM matrix, or finding different processes that require different values for the same matrix element when it is assumed that only standard model interactions contribute to each of the processes, would be evidence of new physics. The decays studied here, $D \rightarrow K l v$ and $D \rightarrow \pi l v$, depend on $\left|V_{c s}\right|$ and $\left|V_{c d}\right|$. They are the two largest elements in the second row of the CKM matrix and provide a stringent test of unitarity, especially if we can reduce the experimental and theoretical errors. Figure 1 graphically shows the contributions of the error in $\left|V_{c S}\right|$ for both leptonic and semileptonic decays. We see that the error for leptonic decays is somewhat smaller than that for the semileptonic decay. We also see that the error from experiment is dominant for the leptonic decay, but that for the semileptonic decay, the error from the lattice QCD calculation of the decay form factor dominates the error. Thus, it is important to reduce the error coming from lattice QCD. We are studying these decays on ensembles of configurations that use the highly improved staggered quark action (HISQ) [1].

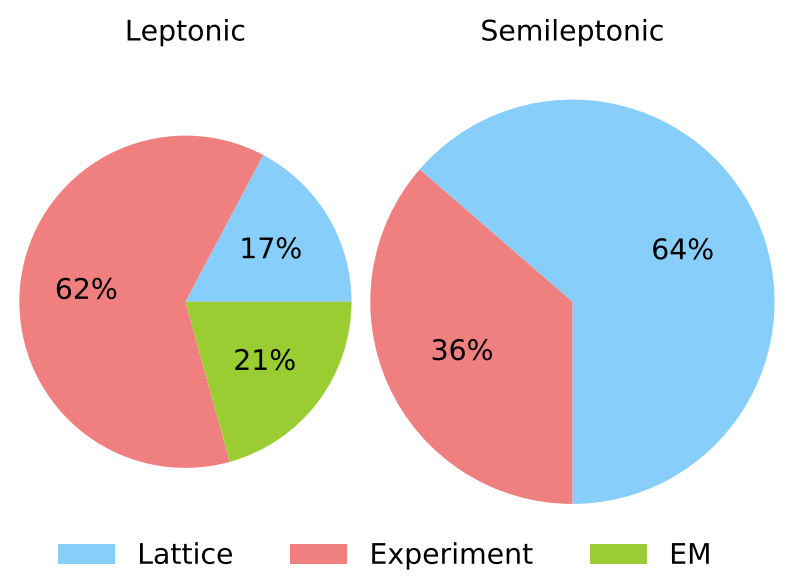

Figure 1: Comparison of contributions to $\left|V_{c s}\right|$ errors from the leading leptonic decay [2, 3] and semileptonic decay $[4,5]$ determinations. Radius is proportional to total error.

\section{Methodology}

To calculate the semileptonic decays of the $D$ meson, we must calculate matrix elements of the vector current operator $V^{\mu}=\bar{q} \gamma^{\mu} c$. We need to calculate $\left\langle K\left|V^{\mu}\right| D\right\rangle$ and $\left\langle\pi\left|V^{\mu}\right| D\right\rangle$. Considering specifically the kaon case, we need $f_{+}\left(q^{2}\right)$ defined through the relation

$$
\left\langle K\left|V^{\mu}\right| D\right\rangle=f_{+}\left(q^{2}\right)\left[p_{D}^{\mu}+p_{K}^{\mu}-\frac{M_{D}^{2}-M_{K}^{2}}{q^{2}} q^{\mu}\right]+f_{0}\left(q^{2}\right) \frac{M_{D}^{2}-M_{K}^{2}}{q^{2}} q^{\mu} .
$$


However, it is more convenient to instead calculate the corresponding matrix element of the local scalar current [6] $S=\bar{q} c$, i.e.,

$$
\left(m_{c}-m_{s}\right)\langle K|S| D\rangle=\left(M_{D}^{2}-M_{K}^{2}\right) f_{0}\left(q^{2}\right)
$$

and use the kinematic constraint $f_{+}(0)=f_{0}(0)$. With staggered quarks, the left-hand side of Eq. (2.2) requires no renormalization, eliminating one potential source of error.

Our calculation makes use of MILC $2+1+1$ flavor HISQ ensembles. The light, strange, and charm valence quarks also use the HISQ action. Figure 2 graphically shows the characteristics of the ensembles that have been used so far. The radius of the inner circle for each ensemble indicates the number of configurations used. The outer radii of the colored circles indicates the product of the number time sources per configuration and number of configurations in each ensemble, i.e., the statistics. The spatial lattice size $L$ is large enough in each case to ensure that $M_{\pi} L>3.5$. The 0.06 fm ensemble with $m_{l}=0.2 m_{s}$ has been added in the past year.

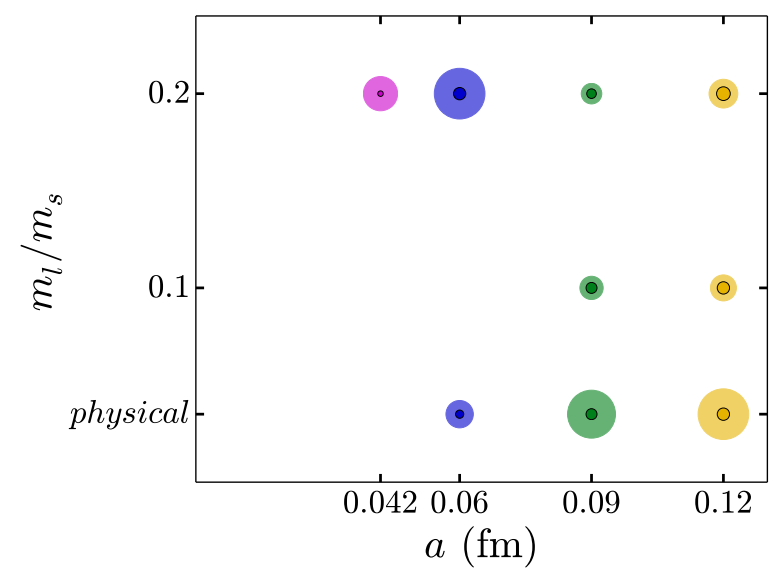

Figure 2: Graphical representation of the ensembles used in this calculation. See text for interpretation of symbol radii.

Using the scalar current and the kinematic constraint, we limit our calculation to $q^{2}=0$. Twisted boundary conditions $[7,8,9]$ are used to calculate three-point functions with an appropriate momentum for the pion or kaon. In general, $q^{2}=M_{K}^{2}+M_{D}^{2}-2 E_{K} M_{D}$. We make use of the dispersion relation $E_{K}=\sqrt{M_{K}^{2}+p^{2}}$ to determine the required momentum to make $q^{2}$ vanish. Momenta up to integer multiples of $2 \pi / L$ come from the usual Fourier transform. The rest comes from a twist of $\theta$ in each spatial direction giving $\vec{p}=\theta \frac{\pi}{L}(1,1,1)$. The momenta required for $q^{2}=0$ are large, and a test of the dispersion relation is shown in Fig. 3. The dashed lines show the expected systematic errors that scale like $\alpha_{s}^{2}(a p)^{2}$. The proportionality constant is set to one to draw the lines. The violations for the kaon are quite small, for the pion, somewhat less so, but the violations appear more random than systematic. (Note the nonmonotonic dependence on $m_{l}$ for $a=0.12 \mathrm{fm}$.) The chiral-continuum fit of our results includes a correction for variations from $q^{2}=0$.

In the proceedings of Lattice 2015 [10], we discussed the quark propagators needed to calculate the three-point functions, and we also explained how we fit the two- and three-point functions. The reader is referred there for further details. The next step in the analysis is the chiral-continuum fit. 

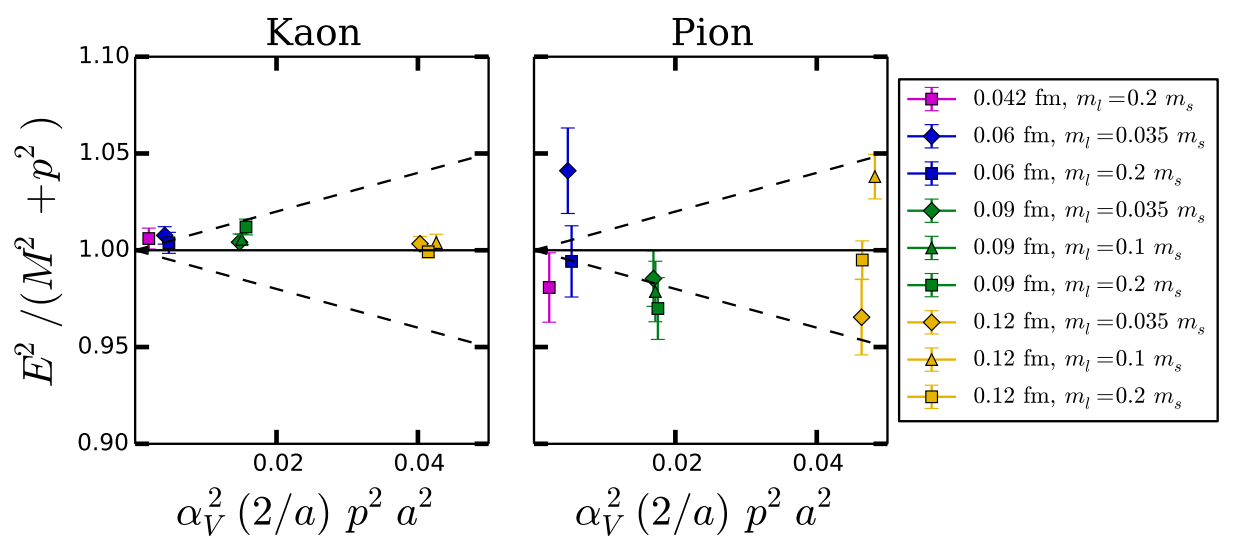

Figure 3: Violation of the energy-momentum dispersion relation for the kaon and pion on each ensemble with the momentum set to the value that would give $q^{2}=0$ assuming the energy-momentum dispersion relation. The strong coupling is defined via the potential, thus the notation $\alpha_{V}$ for the strong coupling.

\section{Chiral-continuum fit, finite volume check, error budget}

We use Heavy Meson Staggered $\chi P T[11,12]$ in the hard pion or kaon limit [13] in order to fit our results. Schematically,

$$
f_{0}\left(q^{2}\right)=\frac{C_{0}}{f_{\pi}}\left[\left(1+\delta f_{\text {logs }}\right)+C_{v} \chi_{v}+C_{s} \chi_{\text {sea }}+C_{a} \chi_{a^{2}}+C_{q} \chi_{q^{2}}\right]
$$

where $\chi_{v}, \chi_{\text {sea }}, \chi_{a^{2}}$ and $\chi_{q^{2}}$ capture the dependence on the daughter quark mass, sea quark masses, light quark discretization effects and $q^{2}$ respectively. We need the value of $g_{\pi}$, the $D^{*}-D-\pi$ coupling, for the chiral logarithms denoted by $f_{\text {logs }}$. We treat it as a fit parameter with a prior of $0.52 \pm 0.07$. The $\chi_{i}$ terms are expected to be multiplied by coefficients $C_{i}$ of order one, so we use priors $C_{i}=$ $0 \pm 2$, for $i \neq 0$. There are additional terms for dependence on $(a p)^{2}, a^{4}$ and other higher order terms that are not included in the central fit, but are included in our systematic error analysis.

Figures 4 and 5 show the chiral-continuum extrapolation for our central fit. In each case, the left panel shows how the data and fit depend on $m_{l} / m_{s}$, and the right panel shows the lattice spacing dependence.

We have explored the systematic error by removing either the dependence on the sea-quark mass, or $q^{2}$ from our central fit. We have also added NNLO terms, an $a^{4}$ term, and an $(a p)^{2}$ term. A stability plot is shown in Fig. 6. The upper green band is the central fit for $f_{+}^{D K}(0)$, and the lower blue band is for $f_{+}^{D \pi}(0)$. The only fit that does not agree very well with the central fit for $f_{+}^{D K}(0)$ is the one for which we removed the sea-quark mass dependence. However, this fit has a p-value of just 0.1 (compared with $>0.5$ for the other fits).

We have looked at three values of the lattice size $L=24,32$, and 40 for $a \approx 0.12 \mathrm{fm}$ and $m_{l}=0.1 m_{s}$, and considered the chiral perturbation theory prediction of Ref. [14] for finite volume effects with twisted boundary conditions. Table 1 contains our error budget. The scale uncertainty comes from adjusting the lattice spacing on each ensemble by $\pm 1 \sigma$. The reported value in the error budget is the largest change observed. 

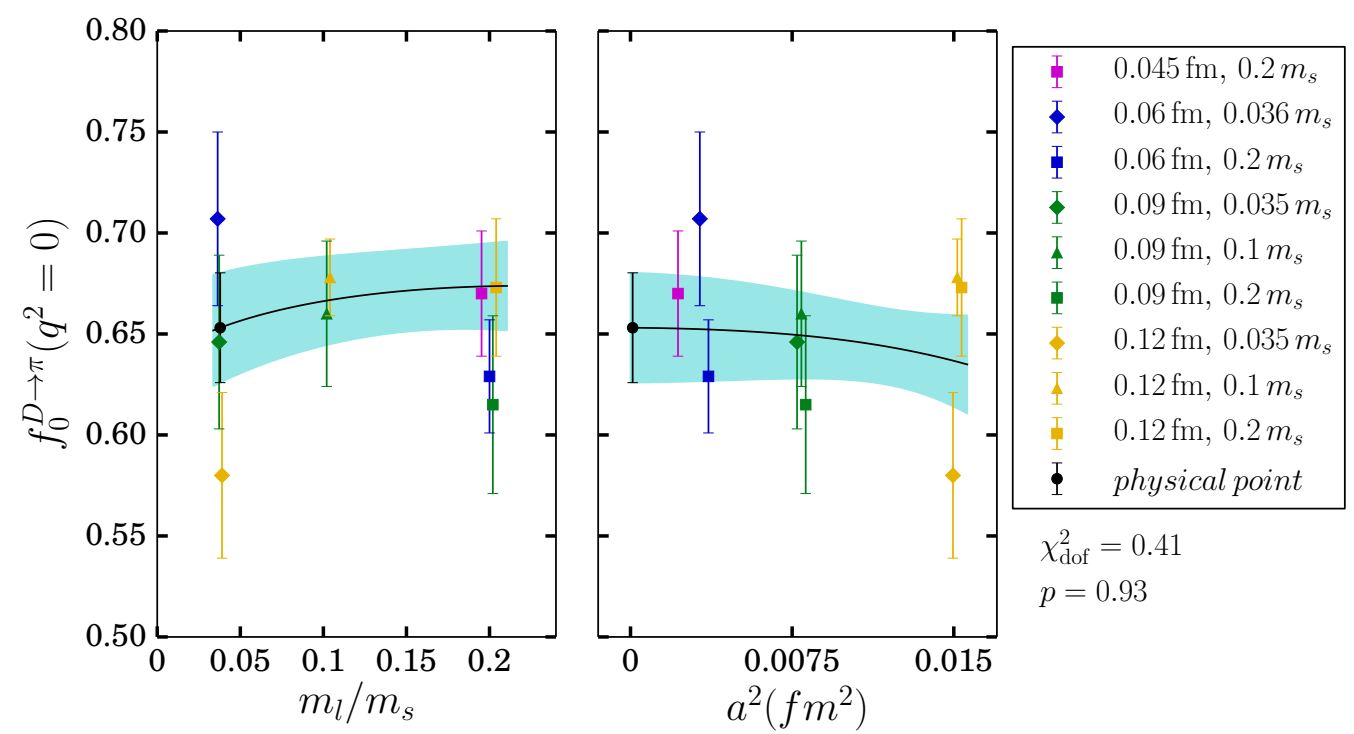

Figure 4: The chiral-continuum extrapolation for $f_{0}^{D \pi}$. In the left panel, we show the dependence on the light quark mass. In the right panel, we show the dependence on $a^{2}$. These are from the same fit, just different projections. The physical value is shown in black.
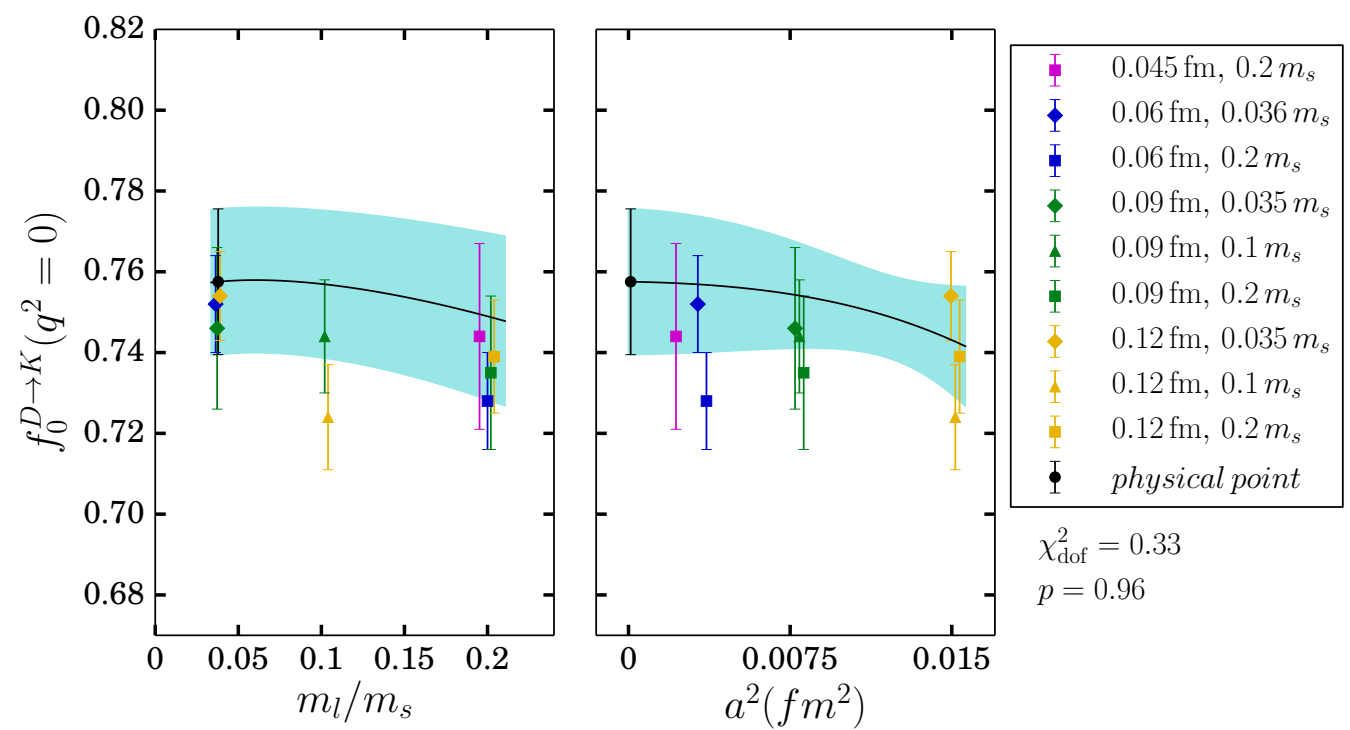

Figure 5: The chiral-continuum extrapolation for $f_{0}^{D K}$.

\section{Conclusions}

In this preliminary report, we have looked at both $f_{+}^{D \pi}(0)$ and $f_{+}^{D K}(0)$ in order to extract the CKM matrix elements $\left|V_{c d}\right|$ and $\left|V_{c s}\right|$, respectively. Although we are not ready to present the values of the form factors, we have done sufficient analysis to anticipate the error budget and final errors. We expect the total error to be $2.4 \%(4.1 \%)$ for $f_{+}^{D K}(0)\left(f_{+}^{D \pi}(0)\right)$, as shown in Fig. 7. Once this analysis is completed, we would like to calculate scalar and vector form factors at multiple values 


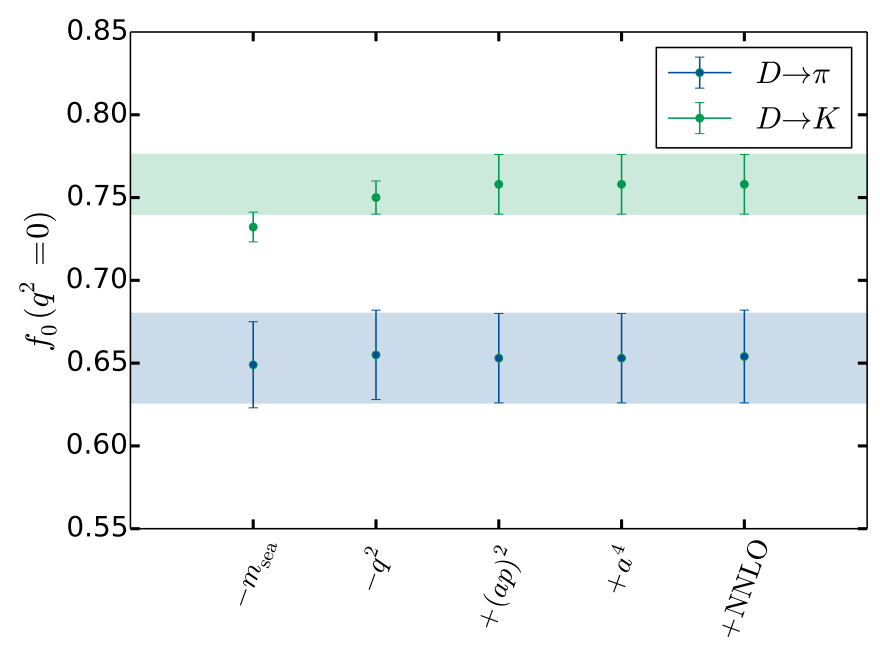

Figure 6: Variation of the chiral continuum limit as we subtract terms from or add terms to the formula used for the central fit.

\begin{tabular}{lcc}
\hline \hline Source of & \multicolumn{2}{c}{$\%$ Error } \\
uncertainty & $f_{+}^{D \rightarrow \pi}(0)$ & $f_{+}^{D \rightarrow K}(0)$ \\
\hline Chiral fit & 4.1 & 2.4 \\
Finite volume & 0.06 & 0.06 \\
Scale $a$ & 0.2 & 0.2 \\
\hline Total & 4.1 & 2.4 \\
\hline \hline
\end{tabular}

Table 1: Preliminary error budget. The chiral fit error includes statistics, the truncation of the chiral model, and discretization errors.

of $q^{2}$. This would allow us to use the $z$-expansion to get the shape and normalization of the form factors across their entire kinematic range, which should reduce the error in the determination of the two CKM matrix elements.

\section{Acknowledgments}

Computations for this work were carried out with resources provided by the USQCD Collaboration; by the ALCF and NERSC, which are funded by the U.S. Department of Energy (DOE); and by NCAR, NCSA, NICS, TACC, and BlueWaters, which are funded through the U.S. National Science Foundation (NSF). Fermilab is operated by Fermi Research Alliance, LLC, under Contract No. DE-AC02-07CH11359 with the US DOE. We acknowledge support by U.S. DOE under grants DE-FG02-91ER40628 (C.B.), DE-SC0010120 (S.G.), DESC0010005 (E.T.N.), DEFG02-13ER42001 (D.D., A.X.K.), DE-FG02-13ER41976 (D.T.) and the U.S. NSF under grants PHY1414614 (C.D.), PHY14-17805 (D.D., J.L.), and PHY13-16748, PHY16-20625 (R.S.). E.G. thanks the MINECO and Junta de Andalucía. A.S.K. thanks the German Excellence Initiative and the European Union Seventh Framework Program under grant agreement No. 291763 as well as the European Union's Marie Curie COFUND program. 


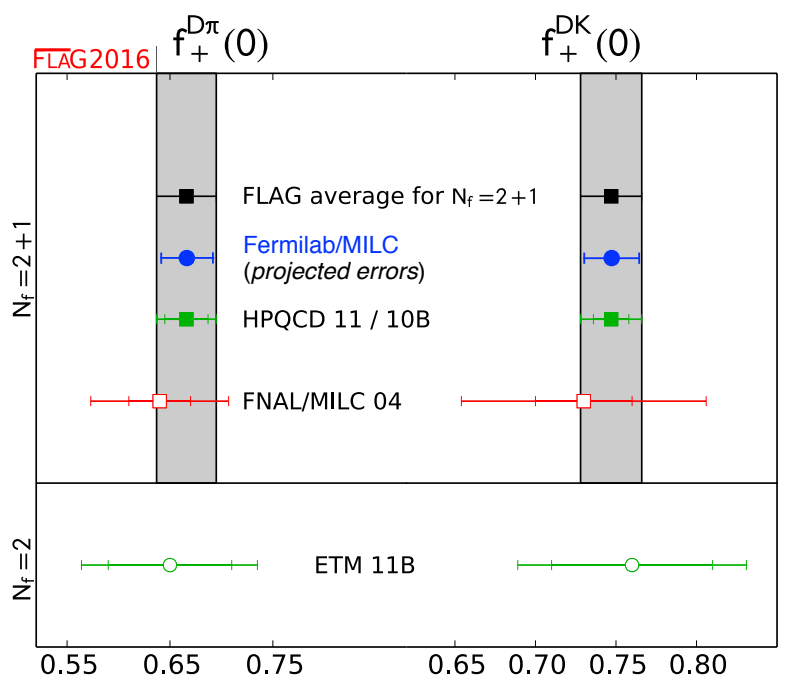

Figure 7: Comparison of our anticipated errors in the form factors for $N_{f}=2+1+1$ with the current results summarized by FLAG for $N_{f}=2$ and $2+1$. The central value from FLAG is used for comparison purposes.

\section{References}

[1] E. Follana et al. [HPQCD and UKQCD Collaborations], Phys. Rev. D 75, 054502 (2007) [hep-lat/0610092].

[2] A. Bazavov et al. [Fermilab Lattice and MILC Collaborations], Phys. Rev. D 90, no. 7, 074509 (2014) [arXiv:1407.3772 [hep-lat]].

[3] J. L. Rosner, S. Stone and R. S. Van de Water, [arXiv:1509.02220 [hep-ph]].

[4] J. Koponen, et. al (HPQCD Collaboration) [arXiv:1305.1462v1],

[5] Y. Amhis et al. [Heavy Flavor Averaging Group (HFAG) Collaboration], arXiv:1412.7515 [hep-ex].

[6] H. Na et al., Phys. Rev. D 82, 114506 (2010) [arXiv:1008.4562 [hep-lat]].

[7] P. F. Bedaque and J. W. Chen, Phys. Lett. B 616, 208 (2005) [hep-lat/0412023].

[8] C. T. Sachrajda and G. Villadoro, Phys. Lett. B 609, 73 (2005) [hep-lat/0411033].

[9] A. Bazavov et al., Phys. Rev. Lett. 112, no. 11, 112001 (2014) [arXiv:1312.1228 [hep-ph]].

[10] T. Primer et al. [Fermilab Lattice and MILC Collaborations], PoS LATTICE 2015, 338 (2016) [arXiv:1511.04000 [hep-lat]].

[11] C. Aubin and C. Bernard, Phys. Rev. D 76, 014002 (2007) [arXiv:0704.0795 [hep-lat]].

[12] D. Becirevic, S. Prelovsek and J. Zupan, Phys. Rev. D 68, 074003 (2003) [hep-lat/0305001].

[13] J. Bijnens and I. Jemos, Nucl. Phys. B 840, 54 (2010) Erratum: [Nucl. Phys. B 844, 182 (2011)] [arXiv:1006.1197 [hep-ph]].

[14] J. Bijnens and J. Relefors, JHEP 1405, 015 (2014) [arXiv:1402.1385 [hep-lat]]. 